\title{
An Efficient Method Development for Extraction of Faecal Volatile Organic Compounds for GC-MS Analysis
}

\author{
Ahmed $I^{1,4 *}$, Zafar Niaz ${ }^{2}$, Cheema $S^{3}$ and Freddie Ewbank ${ }^{4}$ \\ ${ }^{1}$ College of Medicine, Al-Faisal University. Riyadh, KSA \\ ${ }^{2}$ Mayo Hospital Lahore Pakistan, Pakistan \\ ${ }^{3}$ International Medical Centre, KSA \\ ${ }^{4}$ University of Southampton UK
}

*Corresponding author: Iftikhar Ahmed, Consultant Gastroenterologist \& Associate Professor Alfaisal University, Aldara hospital and Medical Center, Riyadh KSA, PO Box 1105, Riyadh 11431 KSA

\section{ARTICLE INFO}

Received: 幽 October 20, 2019

Published: 幽 October 29, 2019

Citation: Ahmed I, Zafar Niaz, Cheema S, Freddie Ewbank. An Efficient Method Development for Extraction of Faecal Volatile Organic Compounds for GC-MS Analysis. Biomed J Sci \& Tech Res 22(2)2019. BJSTR. MS.ID.003736.

Keywords: Volatile Organic Compounds; Gas Chromatography-Mass Spectrometer; Methodology

\begin{abstract}
The study of volatile organic compounds (VOCs) in clinical diagnosis has gained significant scientific interests in the recent decade. Research investigating the potential of these volatile compounds as diagnostic biomarkers of disease is expanding. However, there is a wide range of variability observed in the results of these studies due to multiple confounding factors caused by physiological and environmental effects, and issues related to sample preparation methodologies. Sample preparation techniques for extracting VOCs from biological specimens are complex, as each step is critical for obtaining precise and reproducible results. Despite the development of sophisticated analytical technologies, various challenges exist in the extraction methods of VOCs from biological fluids due to a multitude of parameters and complex nature of analytes. A robust methodology for sample preparation is therefore required for enhanced sensitivity and reproducibility of the results. In this study, we investigated the important steps involved in the sample preparation of faecal aliquots and VOCs extraction from the headspace of these faecal aliquot in order to develop an efficient method.
\end{abstract}

Abbreviations: IBD: Inflammatory Bowel Disease; SPME: Solid Phase Micro Extraction; PDMS: Polydimethylsiloxane; PDMS-CAR: Polydimethylsiloxane Carboxane; PDMS-DVB: Polydimethylsiloxane with Divinylbenzene; CD: Crohn Disease; UC: Ulcerative Colitis

\section{Introduction}

Human biological excretions (faeces, urine, breath sweat etc.) contain a complex mixture of volatile organic compounds (VOCs), which are produced as a by-product of metabolic processes within the body. These VOCs are spread across a wide range of polarity, vapour pressure variation and molecular weight. Analysis of such a complex mixture may pose various challenges and require several steps e.g., separation of these VOCs from the matrix, quantification and analysis using different analytical techniques. Each step is critical for obtaining precise and reproducible results. Although the development of modern analytical instruments allows great enhancement in various aspects of analysis, in many cases the available analytical instrumentation does not have enough sensitivity for the analysis of biological specimens. Sample preparation is still a bottleneck for overall throughputs because the steps involved often employ large volumes of hazardous organic solvents are time-consuming and expensive. In addition, there are issues of contamination and sample loss. There is no single method which can extract effectively all the compounds that may be present in the headspace of analyte due to the complex nature of these VOCs, high-water content of the headspace and the likelihood of some compounds having similar retention time. Solid phase micro extraction (SPME) has been considered a popular sample extraction technique prior to GC-MS analysis of VOCs. It was first described by Pawlisyzn in 1989 [1] and has been increasingly popular 
ever since due to its advantages of reduced solvent consumption, smaller sample volume, automation and shorter analysis time with high precision.

SPME is a fibre coated with either a liquid (polymer), or a solid (sorbent) or combination of both. The small volume of extraction phase allows for the combination of sample preparation and sample introduction into one step. The fibre coating extracts the VOCs from a sample by the process of absorption in case of liquid coatings, and adsorption in case of solid coatings. The analysis of biological specimens using this technique generally involves three steps; pre-concentration of VOCs by heating the aliquot for a certain length of time, and then extraction of these VOCs using
SPME fibre injected into the headspace above the matrix. The SPME fiber is then transferred, with the help of a syringe-like device, to the analytical device (such as GC-MS) for desorption and analysis of the target analytes [2]. The whole process is shown in a schematic diagram below (Figure 1). Previous studies have shown that certain parameters such as vial volume, type of SPME fibre and quantity of faecal sample can have effect on the number and abundance of VOCs extracted from these samples [3]. In this study, we evaluated these important parameters involved in the preparation of faecal sample for VOCs extraction using SPME. The parameters studied were, fibre selection, extraction time, sample quantity and duration between sample production, preparation and analysis.

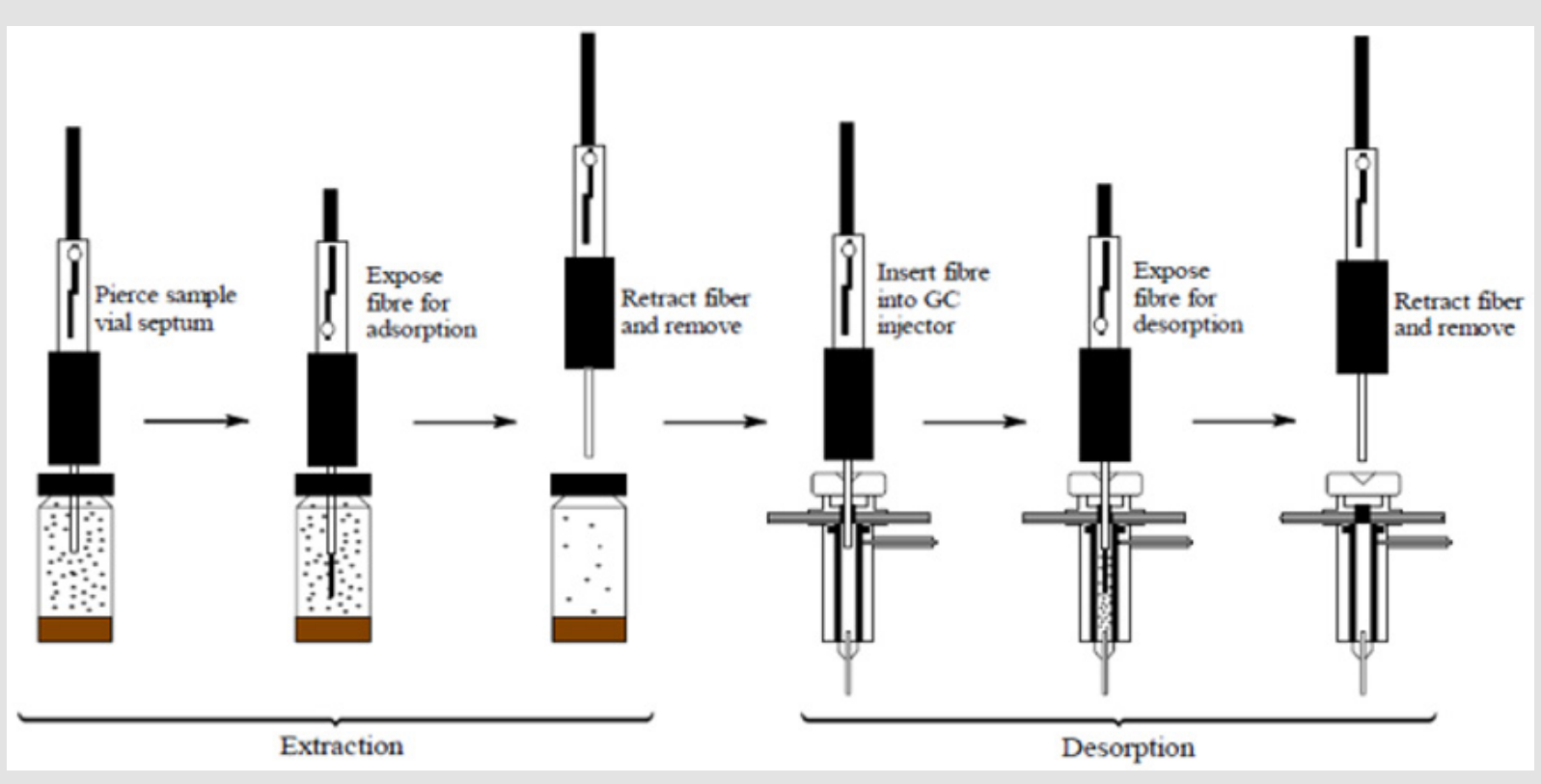

Figure 1: A schematic diagram of faecal VOC extraction and analysis using SPME coupled with GC-MS. Adapted from from Journal of Chromatography A, 880, Kataoka H, Lord LH, Pawliszyn J, Applications of solid-phase microextraction in food analysis, page 40, Figure 3, Copyright (2000), with permission of Elsevier [2].

\section{Material and Method}

A group of healthy volunteers donated faecal samples which were collected in $50 \mathrm{mls}$ faecal sample collection bottles (Biomedics, Cheshire UK) at the room temperature. Samples were homogenised and were aliquoted in an air tight 18mls glass vial (Supelco, Sigma Aldridge UK). Aliquots were either analysed as fresh or stored in the freezer at $-20^{\circ} \mathrm{C}$ after preparation. For analysis, the samples were heated in a thermostatted water bath at $60^{\circ} \mathrm{C}$ for one hour prior to extraction with SMPE fibre injected into the vial headspace above the faecal specimen. The fibre was then transferred to the injection port of GC/MS for analysis. The VOCs were thermally desorbed by immediately transferring the fibre into the heated injection port $\left(220^{\circ} \mathrm{C}\right)$ of a Clarus 500 (Perkin Elmer, Beaconsfield UK) GC-MS. The injector was operated in splitless mode and fitted with a $1.5 \mathrm{~mm}$ i.d. liner. The GC was fitted with a SPB-1 column $(60 \mathrm{~m} \times 0.25 \mathrm{~mm}$ i.d, 1 -micron thick stationary phase, Supelco, UK). The injector was equipped with a $1.5 \mathrm{~mm}$ quartz liner and operated in splitless mode. The oven temperature was held at $40^{\circ} \mathrm{C}$ for 2 minutes after the injection, and then heated up at $6^{\circ} \mathrm{C} /$ minute to $220^{\circ} \mathrm{C}$ and held for 4 minutes giving a run time of 36 minutes. Pure helium (99.95\%, BOC, Guilford, UK) was used as the carrier gas at a constant linear velocity of $35 \mathrm{~cm} / \mathrm{sec}$.

The MS was operated in electron ionization mode scanning a mass range 17-350 with the filament emission current set at $200 \mathrm{~mA}$. The ionization waveform was set 'on'. The ion trap was operated at a target value of 50, a trap offset of $10 \mathrm{~V}$ and at a sampling rate of 2 scans/sec. The multiplier was set at 3.96105. The ion trap manifold temperature was set at $180^{\circ} \mathrm{C}$ and the transfer line was $220^{\circ} \mathrm{C}$. Ethanol standards (50ppm, BOC, Guilford, UK) were used to ensure the SPME fibre efficiency daily. Each chromatogram generated was analysed for identification of compounds. The chromatograms were integrated and a search criterion for peak identification was set at peak area of 1,000,000. Faecal VOCs were identified by comparing the fragment pattern with those in the National Institute 
of Standard and Technology 2008 (NIST) library with a set match criteria of more than $90 \%$ followed by manual visual inspection using retention time matching of selected standards (Fisher Scientific, Loughborough, UK; Acros Organics, London, UK; SigmaAldrich, UK) where needed. There were small numbers of peaks for which the NIST library search did not find a correct match. This was either because the compound was not in the NIST library or may be because two or more different compounds co-eluted making correct assignation very challenging. Such unidentified peaks were named as unknown compounds as per their retention time (for example "unknown compound RT-30.8" for peak appeared at 30.8 minutes) and these unknown compounds were also included in the analysis as unknown compounds. All chromatograms were re-inspected for the presence of sub-threshold peaks and compounds were recorded where a match was available after background subtraction.

The parameters which were investigated in this study were:

a. Time interval of $<2 \mathrm{~h}$, vs. $6 \mathrm{~h}$ vs. $>6 \mathrm{~h}$ between sample production and preparation

b. Sample quantity of $1 \mathrm{gm}$ vs. $2 \mathrm{gm}$ vs. $4 \mathrm{gm}$

c. Polydimethylsiloxane Carboxene (PDMS-C) vs. Diviniylebenzene (DVB) coated SPME fibers d. Extraction time, $10 \mathrm{~min}$ vs $20 \mathrm{~min}$.

e. Comparison of samples analysed fresh vs frozen for 1week, 6 weeks and 6 months

In each set of experiments, only one parameter was studied at a time keeping the rest of the experimental conditions identical.

\section{Results}

\section{Analysis- 1}

In this set of experiments, five volunteers (V1-V5) donated fresh faecal samples in $50 \mathrm{ml}$ faecal sample collection bottle (Biomedics, Cheshire UK). Faecal specimens from each individual were aliquoted into 2 separate vials (Supelco, Sigma Aldridge UK) in equal quantity (2gm each) and were analysed using two SPME coatings (PDMS-C and DVB), keeping the rest of the experimental conditions identical. The results of comparison analysis showed that PDMS-C coated SPME fibre extracted V1=58, V2=80, V3=58, V4=66 and V5=58 VOCs in comparison to DVB coated SPME fibre which extracted $\mathrm{V} 1=46, \mathrm{~V} 2=57, \mathrm{~V} 3=42, \mathrm{~V} 4=58$, and V5=46 VOCs $(\mathrm{p}<$ 0.05). More numbers of VOCs were extracted by PDMS-C coating than the DVB coated fibre in all five sets of experiments. A graph to compare each fibre extraction ability is shown in Figure 2.

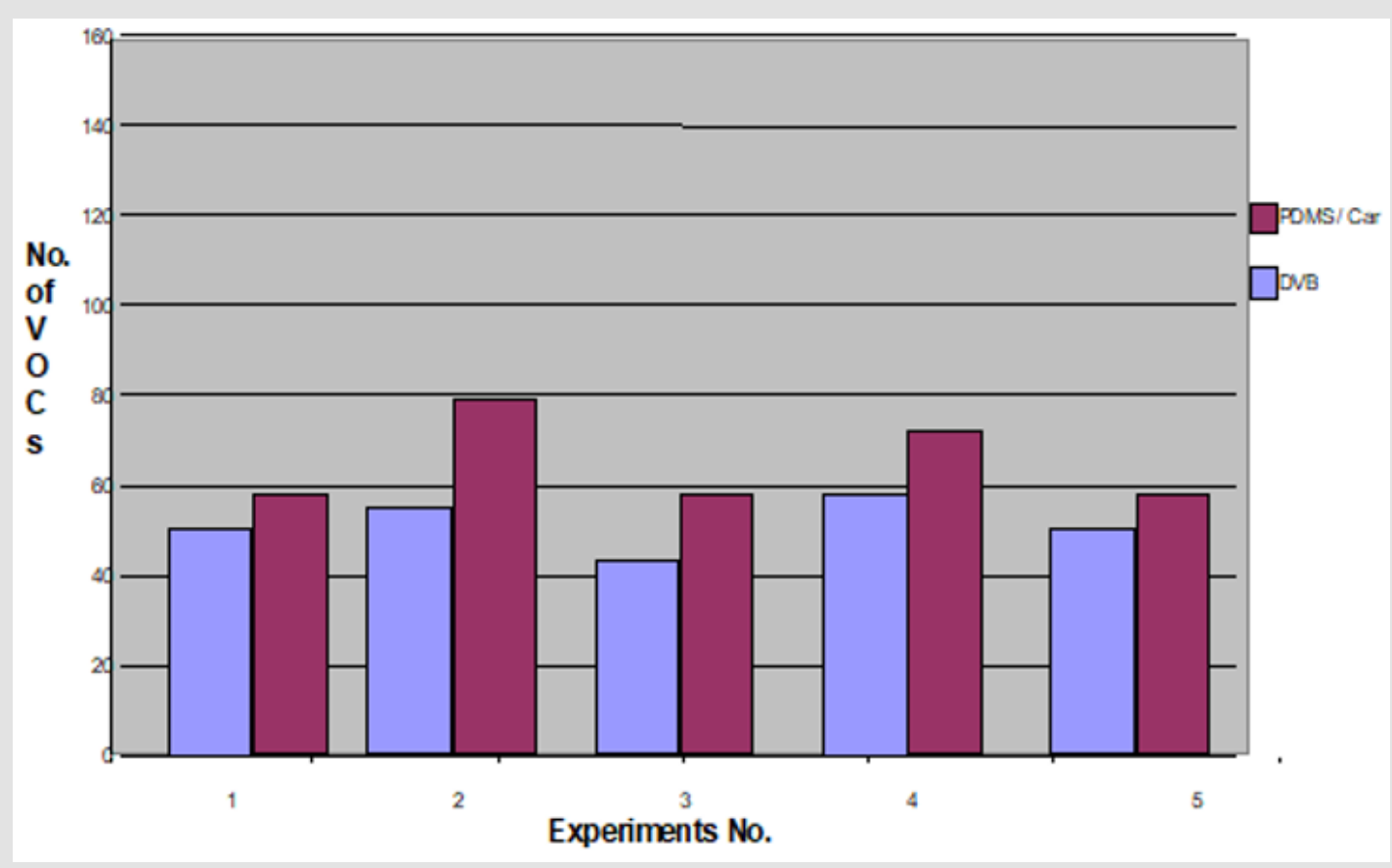

Figure 2: The results of experiments performed to compare the extraction capability of two SPME fibres, PDMS-C vs. DVB. Note: Comparison of PDMS/Ca with DVB fibre

\section{Analysis-2}

In this set of experiments, three volunteers (V1-V3) donated fresh faecal samples in $50 \mathrm{ml}$ faecal sample collection bottle (Biomedics, Cheshire UK). Faecal specimens from each individual were aliquoted into 7 separate $18 \mathrm{ml}$ vials (Supelco Sigma Aldridge UK) in equal quantity (2gm each). Four vials of specimen from each individual were left at room temperature for analysis at different time intervals ( $<2 \mathrm{hrs}, 3 \mathrm{hrs}, 6 \mathrm{hrs}, 12 \mathrm{hrs}$ ) from aliquot preparation, while the other three vials were frozen at $-20^{\circ} \mathrm{C}$ immediately after sample preparation to be analysed at different time intervals ( 1 week, 6 weeks, 6 months). The VOCs were extracted using PDMS-C coated SPME fibre keeping the rest of experimental and sample 
preparation conditions identical. The results showed that more VOCs were identified when fresh samples were analysed within 2 hours of fresh samples preparation (V1=76, V2=84, V3=98 VOCs respectively) compared to when analysed at $3 \mathrm{hrs}(\mathrm{V} 1=70$, $\mathrm{V} 2=80, \mathrm{~V} 3=92 \mathrm{VOCs}), 6 \mathrm{hrs}(\mathrm{V} 1=66, \mathrm{~V} 2=78, \mathrm{~V} 3=86 \mathrm{VOCs})$ and 12 hrs (V1=40, V2=58, V3=54 VOCs). Overall, there was minimal loss of VOCs when fresh specimens were analysed within 6 hours of sample production $(\mathrm{p}<0.01)$.

Fewer VOCs were extracted at 12 hours after sample production showing marked loss of VOCs when samples were analysed beyond 6 hours of sample production. A graph of total number of VOCs detected in all three experiments of each group (i.e. less than 2 hours, 3 hours, 6 hours and 12 hours) is shown in the graph (Figure 3) below. The aliquots which were frozen immediately after sample preparation were thawed and analysed at different intervals (1 week, 6 weeks and 6 months). The results revealed that analysis at 1 week extracted V1 $=68, \mathrm{~V} 2=76, \mathrm{~V} 3=88 \mathrm{VOCs}$, at 6 weeks extracted $\mathrm{V} 1=66, \mathrm{~V} 2=70$ and $\mathrm{V} 3=88$ VOCs and at 6 weeks extracted $\mathrm{V} 1=62$, $\mathrm{V} 2=68$ and $\mathrm{V} 3=86 \mathrm{VOC}$ respectively $(\mathrm{p}<0.01)$. The results of fresh aliquots at different time intervals and those which were initially frozen and then thawed and analysed at different time intervals are shown in the graphs below (Figure 4).

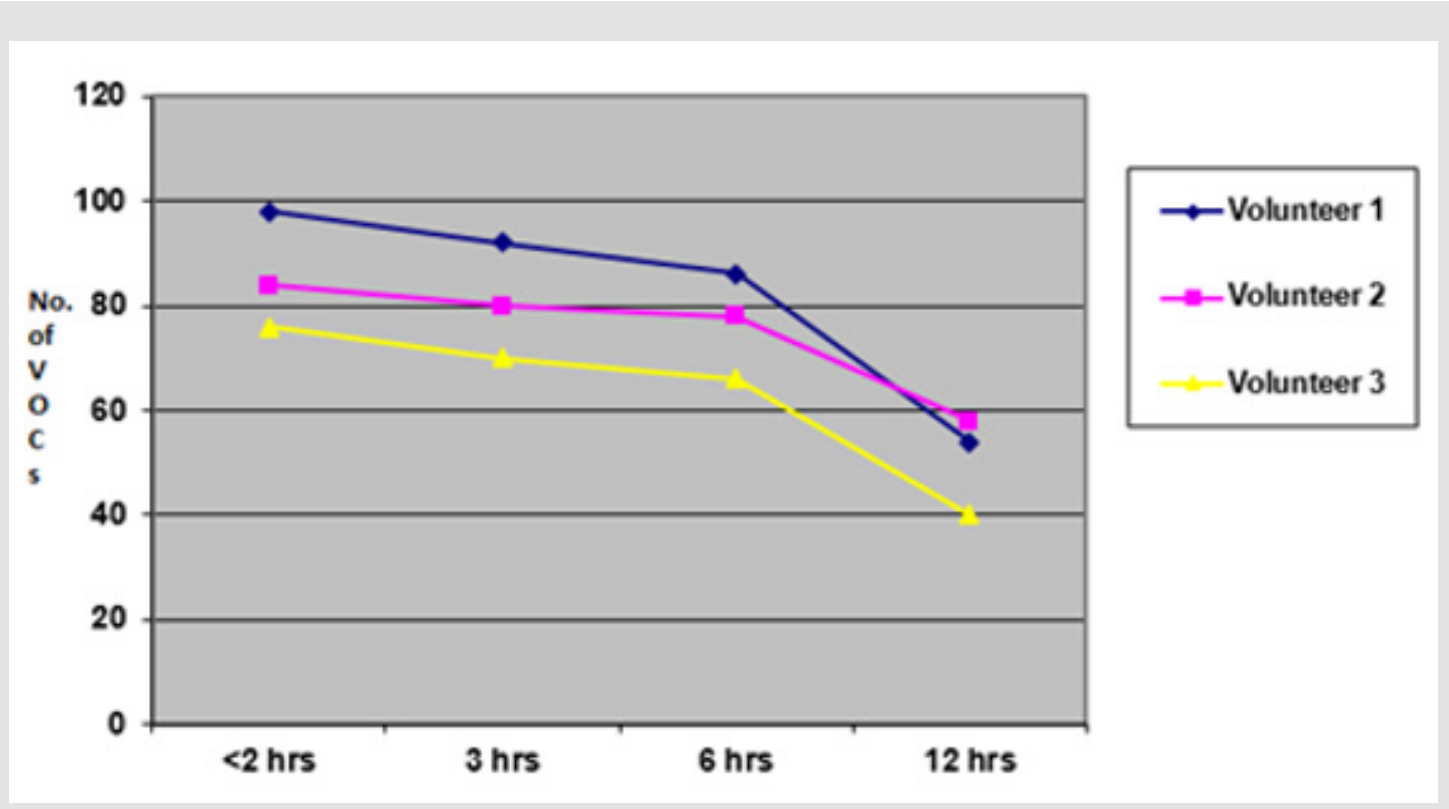

Figure 3: Graph showing total number of VOCs detected from faecal samples analysed at $<2$ hours, 3 hours, 6 hours and 12 hours times from sample productions.

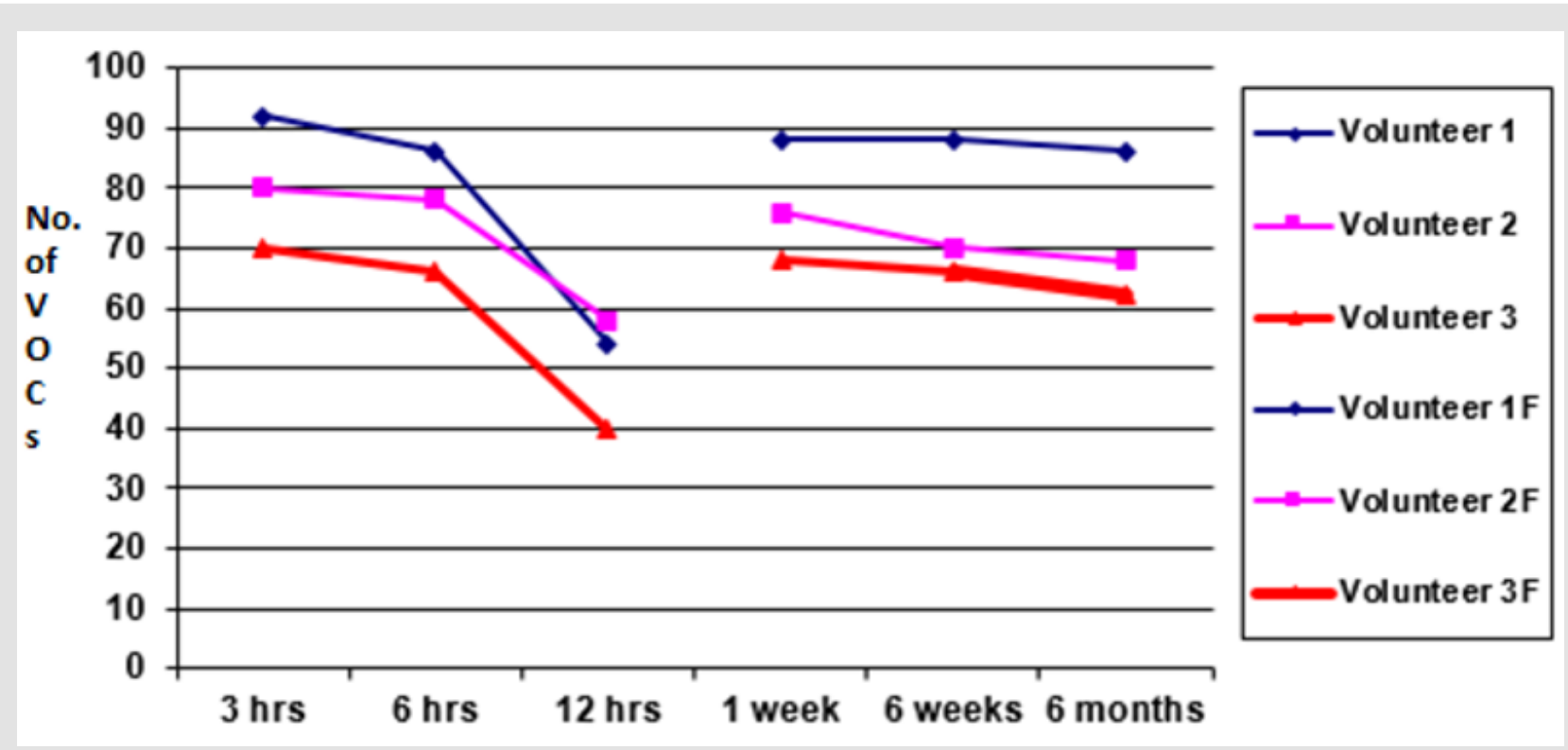

Figure 4: Graph showing an average number of VOCs detected from faecal samples stored at room temperature for $3 \mathrm{~h}, 6 \mathrm{~h}$ and $12 \mathrm{~h}$. The second graph lines are comparison of VOCs detected from samples stored at -20 for 1 week, 6 weeks 6 months. 


\section{Analysis-3}

In this comparison, five volunteers (V1-V5) donated fresh faecal samples in $50 \mathrm{ml}$ faecal sample collection bottle (Biomedics, Cheshire UK). Faecal specimen from each individual were aliquoted into 3 separate vials (Supelco Sigma Aldridge UK) in different quantity (1gm, 2gm and 4gm each). The VOCs were extracted using
PDMS-C coated SPME fibre keeping the other experimental conditions identical. The results of these experiments revealed that more VOCs were extracted from the $2 \mathrm{gm}$ aliquots $(\mathrm{V} 1=61, \mathrm{~V} 2=92, \mathrm{~V} 3=50$, $\mathrm{V} 4=60,59$ VOCs) compared to the 1 gm aliquots (V1=51, V2=79, $\mathrm{V} 3=49, \mathrm{~V} 4=57, \mathrm{~V} 5=57 \mathrm{VOCs})$ and $4 \mathrm{gm}$ of aliquots (V1=51, V2=89, $\mathrm{V} 3=46, \mathrm{~V} 4=46, \mathrm{~V} 5=51 \mathrm{VOCs})$ respectively $(\mathrm{p}<0.01)$. The results of these comparisons are shown in the graph below (Figure 5).

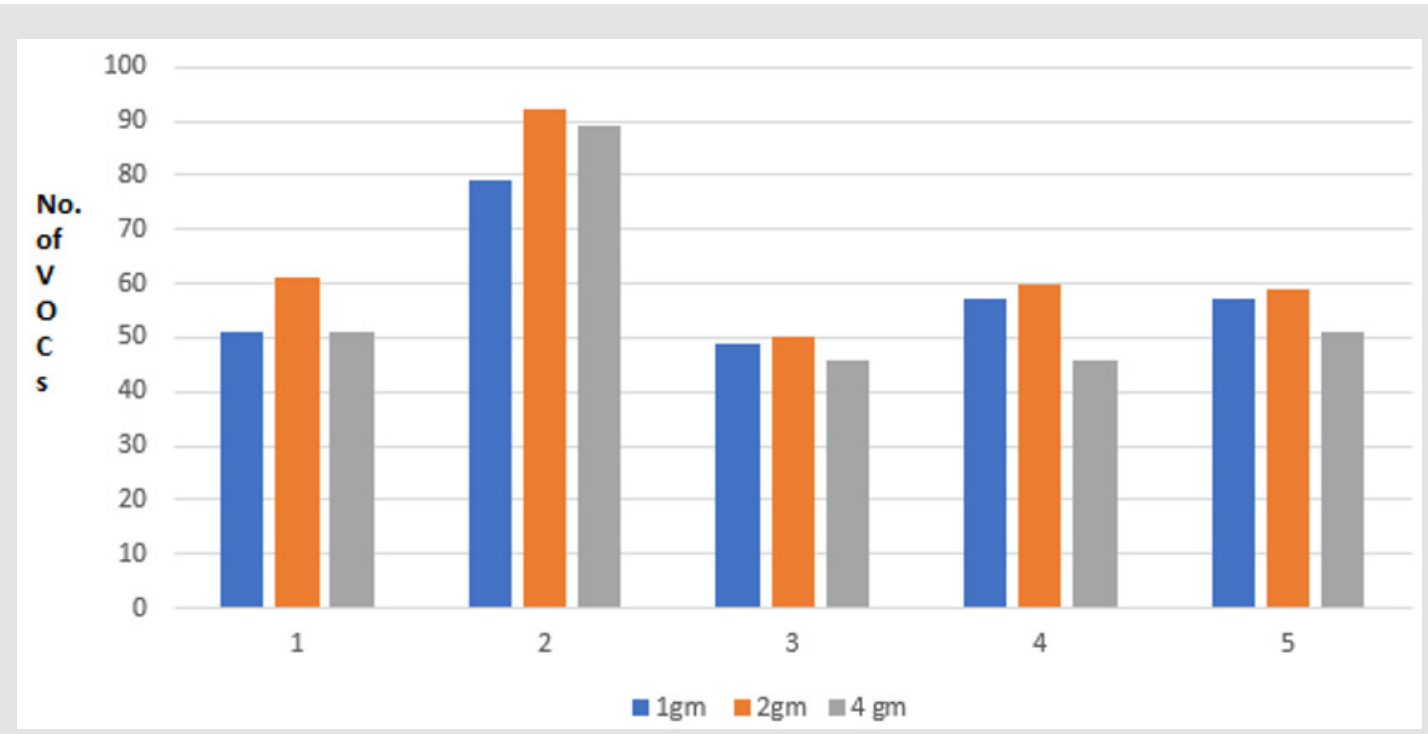

Figure 5: Graph showing total number of VOCs extracted from different quantity of faecal samples, $1 \mathrm{gm}$ vs $2 \mathrm{gm}$ vs $4 \mathrm{gm}$. Note: Comparison of VOCs extraction from different stool quantity

\section{Analysis-4}

In this comparison, five volunteers (V1-V5) delivered fresh faecal samples in $50 \mathrm{ml}$ faecal sample collection bottle (Biomedics, Cheshire UK). $2 \mathrm{gm}$ of faecal specimen from each individual was aliquoted into 2 separate vials (Supelco Sigma Aldridge UK). Fresh aliquots were analysed using PDMS-C coated SPME fibre to compare 10 minutes and 20 min extraction times. The rest of the ex- perimental conditions were kept identical. The results showed that 10 minutes of extraction by SPME fibre extracted more VOCs from three out of five aliquots (V1=62, V2 $=51, V 3=46, V 4=57, V 5=60$ VOCs) compared to 20 minutes extraction $(V 1=58, V 2=52, V 3=64$, $\mathrm{V} 4=51$ and $\mathrm{V} 5=55 \mathrm{VOCs}$ ). In one comparison (V2) the total number of VOCs was not different between the two extraction times and in only one comparison (V3), more VOCs were extracted by 20 minutes extraction $(\mathrm{p}<0.01)$ as shown in the graph below (Figure 6).

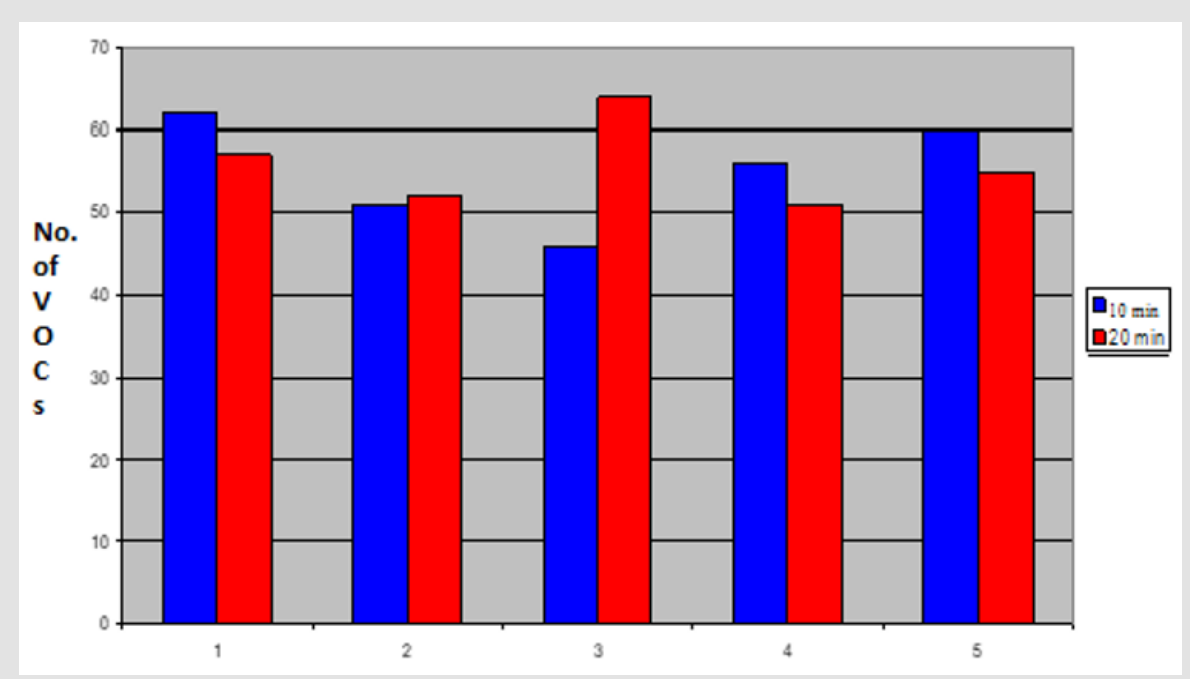

Figure 6: Graph showing total number of VOCs extracted by SPME fibre from faecal samples by using different extraction times 10 minutes vs 20 minutes. 


\section{Discussion}

Sample preparation remains an essential step in any biomedical analysis; it can greatly influence the reliability and accuracy of the results, the time required for analysis and overall cost [4]. It generally involves extracting the components of interests from the sample matrix prior to the transfer of these to analytical equipment. Sample preparation can be a very time-consuming, laborious and multi-stage process and varies depending upon the nature of the sample matrix. Various surveys have shown that in any experimental analyses, significant amount of time is spent on sample collection and preparation [5,6]. This is necessary because, in most cases, analytical instruments cannot handle the sample matrices directly. The whole analytical process of faecal VOCs using SPME extraction method and analysis by GC-MS techniques can produce unreliable results if an unsuitable sample preparation method has been employed before the sample reaches the chromatography equipment. Modern techniques offer the advantages of low consumption of reagents, samples and organic solvents, high speed and low cost [7].

The absorption of VOCs from the matrix depends upon two equilibrium stages; firstly, an equilibrium between the matrix and the headspace which is mainly dependent on the volatility of the analytes and physical characteristics of the matrix and, secondly, an equilibrium between the headspace and fibre coating and this depends upon the diffusion of VOCs from headspace to the fiber coating [8]. The extraction of VOCs by the SPME fiber is rapid in the first few minutes which slow down later until equilibrium is reached [9]. The work by Garner et al. [10] determined that 20 min was the optimum time for extraction of VOCs from head-space of faecal aliquots using SPME technique. Other studies also used 20 minutes time for extraction of VOCs from the headspace of faecal aliquot using SPME fibre [11]. Since extraction of VOCs is rapid in the first few minutes, we evaluated whether shorter extraction interval can produce similar results. Our results showed that 10 minutes was an optimum extraction time as approximately equal or slightly more VOCs were extracted from the head space of faecal aliquots compared to 20 min extraction. These results suggest that shorter sample extraction time will reduce the overall duration of the experiment and would have significant implication on the efficiency of laboratory work.

Other important question in sample preparation and extraction method is the choice of SPME fibre coating for an efficient VOCs extraction from the head space. Fibre selection depends upon volatility, polarity and molecular weight of the VOCs. Analysis of a matrix containing a complex mixture of these VOCs will require a fibre which has the capability of extracting VOCs of a wide variety [10]. Two types of SPME coating were tested in the studies with good results [12]. We compared these two SPME coatings in this study and our results showed that PDMS-C fibre coating was better than the PDMS -DVB coating in extracting the wide range of VOCs from human faeces. PDMS/Car fibre was also found to be better in other studies for extracting VOCs from various matrixes compared to other coatings [13]. In a recent study, Hough et al. compared the efficiency of PDMS-C type of SPME fiber coatings with PDMSC-DVB (dual coating with carboxane as well as divinylbenzene) on the extraction of VOCs from equine faecal sample [14]. This study found that Significantly more VOCs were detected with a DVB-CARPDMS fibre than from a CAR-PDMS fibre suggesting that multiple fibre coatings increases the diversity of VOCs obtained from faecal sample. These results were also supported by another study by Read et al. $[3,15]$.

Collection of faecal samples is a challenging issue, and in contrast to blood sampling which can be collected as and when required, faecal sample can only be donated when an individual opens his or her bowel. In clinical practice, patients are usually asked to bring faecal sample from home if they are being seen in the clinic due to ease of sample production and collection within the home privacy $[15,16]$. This usually cause delay in transporting the sample to the laboratory and its analysis. If patients are admitted in the hospital, sample can be collected and transported to the laboratory fairly quickly but there will still be a certain delay of time required for transportation of samples to the laboratory etc. Since VOCs are emitted from faecal matter at room temperature, leaving sample without preservation for longer time would have implications on the number or abundance of VOCs extracted from such samples. It is, therefore, important to analyse the sample without delay to avoid the loss of VOCs. Theoretically, the longer the time between sample production and analysis, the greater this loss would be. Determining this minimum interval of time is important in order to allow time for transportation of the faecal specimen and analysis without compromising the efficacy of the results in clinical practice. However, this issue hasn't received due attention and studies on this are limited [17].

Our results showed that minimum loss of VOCs occurs if fresh sample are analysed during the first 6 hours of sample production. Similarly, storage of faecal sample may be required if a sample needs to be transported to a distant laboratory, or for research purposes (for reproducibility of the results etc). Our results also showed that freezing the sample after production will minimize such loss and samples can be stored for long periods if they are frozen. We observed that samples can be stored for 6 months at -20C without any significant loss of VOCs. The study by Hough et al. compared the extraction of VOC from fresh faecal sample from equine compared to sample preserved at $-20 \mathrm{C}$ or $-80 \mathrm{C}$. Interestingly their results showed that higher number of volatile compounds were identified from faecal sample stored at -20C compared to fresh samples or those stored at -80C. However, majority of compounds identified from sample stored at -20C were esters and it wasn't clear whether the increased number of esters identified were due to the effect of temperature, storage vials or difference in gut physiological 
environment between equine and human. In another study by Gratton et al. on human faeces, it was observed that after $24 \mathrm{~h}$ of sample storage at $-20{ }^{\circ} \mathrm{C}$ there was no difference in VOC profile extracted from fresh sample compared to samples which were initially frozen and then later thawed and analysed [18].

SPME is not an exhaustive extraction; instead equilibrium is established between the gas phase of the headspace and stationary phase of the fibre coating $[19,20]$. The partitioning between the gas phase and the stationary phase can be described through the distribution constant, $\mathrm{K}$

\section{$\mathrm{K}=\mathrm{Cs} / \mathrm{Cg}$}

Where $\mathrm{Cs}$ is the concentration in the stationary phase and $\mathrm{Cg}$ is the concentration in the gas phase.

Once the equilibrium between these two phases is reached, further extraction of VOCs from the gas phase does not occur. If the fibre is left exposed for longer duration, it may actually cause the loss of VOCs from the stationary phase of the fibre. Our results have showed that 10 minutes was the optimum time for extraction of maximum numbers of VOCs from headspace of faeces in a $18 \mathrm{ml}$ sealed vial as number of volatiles extracted in 10 minutes did not differ much from 20 minutes extraction.

\section{Concluding Remarks}

As improvements in technology allow for lower and lower levels of VOCs detection from biological fluids, it is imperative that a proven and sound sample preparation methodology is developed and followed to achieve reliable and consistently reproducible results. Based on our study results, we concluded that the optimal experimental conditions for the extraction of faecal VOCs by SPME technique were $2 \mathrm{gm}$ of faecal specimen aliquoted in $18 \mathrm{ml}$ sealed vial, analysed within 6 hours of sample production, using PDMS/ Car type of SPME fibre exposed to the headspace for 10 minutes. If samples need storage for later analysis, then these should be frozen at -20C to 'preserve' the VOCs within the matrix. These parameters were used in our experiments for the VOCs analysis in order to compare pattern of faecal VOCs of patients with IBD and healthy controls [21]. Further studies are needed in this area to enhance the solidarity of methodology for VOCs analysis. Development of agreed national standards would be a key to minimise inaccuracies and maintain consistency of the results.

\section{Acknowledgement}

The principal authors acknowledge Dr Steve Smith and Dr Catherine Garner for their technical support for acquisition and analysis of laboratory data.

\section{References}

1. Pawliszyn J (2000) Theory of solid phase microextraction. J Chromatogr Sci 38(7): 270-278.

2. Kataoka H, Lord LH, Pawliszyn J (2000) Applications of solid-phase microextraction in food analysis. Journal of Chromatography A 880(12): $40-42$.
3. Reade S, Mayor A, Aggio R, Khalid T, Pritchard D, et al. (2014) Optimisation of sample preparation for direct SPME-GC-MS analysis of murine and human faecal volatile organic compounds for metabolomic studies. Journal of Analytical \& Bioanalytical Techniques 5: 184.

4. Issaq HJ, Van QN, Waybright TJ, Muschik GM, Veenstra TD (2009) Analytical and statistical approaches to metabolomics research. J Sep Sci 32(13): 2183-2199.

5. Saito Y, Jinno K (2003) Miniaturized sample preparation combined with liquid phase separations Journal of Chromatography A 1000(1): 53-67.

6. Ulrich S (2000) Solid phase microextraction in biomedical analysis. J Chromatogr 902(1): 167-194.

7. Ettre LS (2001) The Evolution of Capillary Columns for Gas Chromatography. LCGC 19(1): 48-59.

8. Ai J (2000) Quantitation by SPME before reaching a partition equilibrium. In Pawliszyn J (Eds.)., The Royal Society of Chemistry, Cambridge, USA.

9. (2001) A Practical Guide to Quantitation with SPME. Supelco Bulletin 929 T: 101929, Supelco: Bellefonte, PA, USA.

10. Garner CE, Smith S, Costello B, White P, Spencer R, et al. (2007) Volatile organic compounds from feces and their potential for diagnosis of gastrointestinal disease. FASEB J 21(8): 1675-1688.

11. Hough R, Archer D, Probert C (2018) A comparison of sample preparation methods for extracting volatile organic compounds (VOCs) from equine faeces using HS-SPME. Metabolomics 14(2): 19.

12. Fibre selection guide, Sigma Aldridge UK.

13. Adam M, Juklová M, Bajer T, Eisner A, Ventura K (2005) Comparison of three different solid-phase microextraction fibres for analysis of essential oils in yacon (Smallanthus sonchifolius) leaves. Journal of Chromatography A 1084(1-2): 2-6.

14. Dixon E, Clubb C, Pittman S, Ammann L, Rasheed Z, et al. (2001) Solidphase microextraction and human fecal VOCs metabolome. PloS One 6(4): 18471.

15. Deng C, Zhang X (2008) A simple, rapid and sensitive method for determination of aldehydes in human blood by gas chromatography/ mass spectrometry and solid-phase microextraction with on-fiber derivatization. Rapid Communications in Mass Spectrometry 18(15): 1715-1720.

16. Kleeberg KK, Liu Y, Jans M, Schlegelmilch M, Strees J, et al. (2005) Development of a simple and sensitive method for the characterization of odorous waste gas emissions by means of solid-phase microextraction and GC-MS/olfactometry. Waste Management 25: 872-879.

17. Phillips M (1997) Method for the collection and assay of volatile organic compounds in breath. Analytical Biochemistry 247(2): 272-278.

18. Gratton J, Phetcharaburanin J, Mullish BH, Williams HRT, Thursz M, et al. (2016) Optimized sample handling strategy for metabolic profiling of human feces. Analytical Chemistry 88(9): 4661-4668.

19. Saito Y, Kawazoe M, Hayashida M, Jinno K (2000) Direct coupling of microcolumn liquid chromatography with in-tube solid-phase microextraction for the analysis of antidepressant drugs. Analyst 125: 807-809.

20. Kolb B, Ettre L (1997) Static Headspace-Gas chromatography: Theory and Practice. Wiley-VCH: Winheim.

21. Ahmed I, Greenwood R, Costello B, Ratcliffe N, Probert C (2016) Investigation of faecal volatile organic metabolites as novel diagnostic biomarkers in inflammatory bowel disease. Aliment Pharmacol Ther 43(5): 596-611. 


\section{ISSN: 2574-1241}

DOI: 10.26717/BJSTR.2019.22.003736

Iftikhar Ahmed. Biomed J Sci \& Tech Res

(c) (P) This work is licensed under Creative

Submission Link: https://biomedres.us/submit-manuscript.php

$\begin{array}{ll}\text { BIOMEDICAL } & \text { Assets of Publishing with us } \\ \text { RESEARCHES } & \text { - Global archiving of articles } \\ \text { - Immediate, unrestricted online access } \\ \text { - Rigorous Peer Review Process }\end{array}$

